\title{
The use of a second biopsy from the gastric body for the detection of Helicobacter pylori using rapid urease test
}

Andrew Wong $^{1}$, MBBS, FRCS, Siok Siong Ching $^{1}$, MRCS, MD, Ai Sha Long ${ }^{1}$, MBBS, MRCS

INTRODUCTION The use of an additional biopsy from the gastric body may help improve the detection of Helicobacter pylori during endoscopy. This study aimed to determine whether such an additional biopsy is necessary in routine rapid urease test (RUT), and whether acid suppression and antibiotic therapy affect RUT results.

METHODS Patients recruited had two gastric mucosal biopsies taken - one from the gastric antrum and the other from the gastric body. Each biopsy was placed into separate RUT kits. Information on previous or current use of proton-pump inhibitors, $\mathrm{H} 2$ receptor antagonist, bismuth and antibiotics was obtained. Patients on any of those drugs one week prior to endoscopy were considered to have a positive drug history (PDH).

RESULTS Of the 400 patients recruited, 311 had negative RUTs and 89 had at least one positive RUT. Between the PDH and negative drug history (NDH) groups, there was a significant difference in the distribution of the location of the biopsies that yielded positive RUTs ( $p=0.023$ ). The NDH group had a higher proportion of patients who had positive RUTs for both locations, whereas the PDH group had a higher proportion of patients who had positive RUTs for only one location. CONCLUSION As RUT results are significantly affected by the use of acid suppression and antibiotic therapies, biopsies for RUT should be taken from both the gastric antrum and body to minimise false negative results.

Keywords: gastric body biopsy, Helicobacter pylori, rapid urease test

\section{INTRODUCTION}

Helicobacter pylori (H. pylori) are Gram-negative, flagellate, microaerophilic bacilli. They are an important factor in the aetiology of active chronic gastritis, duodenal ulcers and gastric ulcers, and are present in almost all patients with these diseases. ${ }^{(1)}$ Currently, H. pylori is also accepted to be the cause of gastric carcinoma and gastric mucosa-associated lymphoid tissue lymphoma. ${ }^{(2)}$ The natural history of $H$. pylori gastritis involves the inflammation of the antrum, which eventually progresses to the adjacent corpus, resulting in an atrophic front of advancing injury that leads to a reduction in the acid secretion of the stomach, the eventual loss of parietal cells and the development of atrophy. ${ }^{(3)}$

The diagnosis of $H$. pylori infection is usually based on the results of a rapid urease test (RUT) and the histology of gastric antral biopsies. H. pylori organisms produce abundant urease and the RUT consists of a urea-rich agar gel medium that has a $\mathrm{pH}$ sensitive dye infused in it. If the mucosal biopsy specimen placed in the urea-rich agar gel contains $H$. pylori organisms (i.e. urease would be present in the specimen), hydrolysis of the urea in the gel would occur. This hydrolytic reaction yields carbon dioxide and ammonium ions, resulting in an increase in the $\mathrm{pH}$ of the gel, and hence, a change in the colour of the indicator in the gel. ${ }^{(4)}$

The migration of $H$. pylori from the gastric antrum to the proximal stomach following acid suppression therapy has been reported. ${ }^{(5)}$ In addition, proton-pump inhibitors (PPIs), bismuth and antibiotic therapy have been shown to suppress $H$. pylori ${ }^{(6)}$ and cause false negative results in $13 \mathrm{C}$-urea breath tests. ${ }^{(7,8)}$ These findings show that the ability to detect $H$. pylori is decreased when patients are on certain medications or have undergone suboptimal $H$. pylori eradication therapy. Some authors have thus suggested the use of an additional biopsy from the gastric body to improve the detection rates of $H$. pylori. ${ }^{(9-12)}$ In the present study, we aim to determine whether a second biopsy from the gastric body is necessary in the routine use of RUT for the detection of $H$. pylori, as well as whether acid suppression and antibiotic therapy affect RUT results.

\section{METHODS}

Based on the seroprevalence of $\mathrm{H}$. pylori in the local population, which was reported to be between $13.7 \%$ and $34.3 \%$, ${ }^{(13)}$ the recruitment of 400 patients would result in at least one positive RUT in approximately 100 patients. This number would be sufficient for satisfactory statistical analysis. Therefore, we recruited 400 patients for this prospective cross-sectional study.

From July 2005 to May 2009, 400 consecutive patients who presented for gastroscopy to the principal investigator of the present study were recruited. These patients needed to undergo gastroscopy to obtain: (a) endoscopic evidence of gastritis, duodenitis or peptic ulcer disease; and (b) a gastric biopsy for the detection of $H$. pylori using RUT. Patients who had previously been treated with $H$. pylori eradication therapy but still presented with nonhealing gastroduodenal lesions were eligible for inclusion in the present study if they had to be retested for the presence of $H$. pylori. Patients who had coagulopathy, did not require $H$. pylori detection testing, or had 
recently been tested for $H$. pylori by methods other than RUT (e.g. urea breath test or $H$. pylori stool antigens) were excluded from the present study. Children aged 12 years and below were also excluded.

The RUT kits (HpFast; GI Supply, Philadelphia, PA, USA) used in the present study had a sensitivity of $88 \%$ and specificity of 99\% for the detection of H. pylori. ${ }^{(14)}$ All patients who met the study requirements had two gastric mucosal biopsies taken one from the gastric antrum and the other from the gastric body. Separate RUT kits were used for each biopsy specimen. The kits were labelled accordingly, and read at 15 minutes, 1 hour and 24 hours after the insertion of the biopsy into the urea agar gel. The results of the RUTs were recorded.

Information on whether the patients included in the present study had previous or current use of PPI, $\mathrm{H} 2$ receptor antagonist, bismuth and antibiotics was obtained. Any previous history of H. pylori eradication therapy was also noted and recorded. Patients were deemed to have a positive drug history $(\mathrm{PDH})$ if they had been on any of the aforementioned groups of drugs within the one week prior to endoscopy. Patients who had not been taking any of those medications within the one week prior to endoscopy were considered to have a negative drug history $(\mathrm{NDH})$, even if they had been taking them previously.

Statistical analysis was performed using IBM SPSS Statistics for Windows version 19.0 (IBM Corp, Armonk, NY, USA). We used chi-square test to determine the relationship between the prevelance of $H$. pylori in various parts of the stomach and the patients' drug history. A p-value $<0.05$ was considered statistically significant. This study was approved by the local ethics committee (Reference no. 2005/12).

\section{RESULTS}

Table I shows the demographics and clinical characteristics of the 400 patients who were recruited for the present study. Out of the 400 patients, 311 (77.8\%) had negative RUT results from both biopsy locations. There was a high prevalence of $\mathrm{PDH}(52.1 \%)$ in the group with negative RUT results from both biopsy locations; these 311 patients were excluded from further analysis. The remaining $89(22.3 \%)$ patients had at least one positive RUT result.

When we compared the patients in the $\mathrm{PDH}$ group with those in the $\mathrm{NDH}$ group, there was a significant difference in the distribution of locations at which RUT was positive $(p=0.023)$. The proportion of patients who had positive RUTs for both locations was higher in the NDH group, whereas the proportion of patients who had positive RUTs for only one location (i.e. either the gastric antrum or body) was higher in the PDH group (Table II).

Based on the data collected, if the biopsies for the RUTs were taken from only the gastric antrum, there would be a $11.5 \%$ ( 6 out of 52) chance and a $29.7 \%$ (11 out of 37 ) chance that a false negative result will be obtained in the $\mathrm{NDH}$ and the $\mathrm{PDH}$ groups, respectively (Table II). The false negative rate in the $\mathrm{PDH}$ group was significantly higher than that in the NDH group $(p=0.031)$. The overall rate of false negative RUTs was $19.1 \%$ (17 out of 89 ).
Table I. Demographic and clinical characteristics of patients $(n=400)$.

\begin{tabular}{lc}
\hline Parameter & No. of patients (\%) \\
\hline Age* (yr) & $49.5 \pm 15.7$ \\
Gender & \\
Male & $229(57.3)$ \\
Female & $171(42.8)$ \\
Ethnicity & \\
Chinese & $289(72.3)$ \\
Malay & $62(15.5)$ \\
Indian & $31(7.8)$ \\
Others & $18(4.5)$ \\
Patient setting & \\
Outpatient & $326(81.5)$ \\
Inpatient & $74(18.5)$ \\
Symptoms & \\
Dyspepsia & $124(31.0)$ \\
Epigastric pain & $228(57.0)$ \\
Bleeding & $43(10.8)$ \\
Others & $104(26.0)$ \\
On acid suppression or antibiotic therapy & \\
No & $201(50.3)$ \\
Yes & $199(49.8)$ \\
Previous H. pylori eradication & \\
No & $370(92.5)$ \\
Yes & $30(7.5)$ \\
Gastritis & \\
Not present & $15(3.8)$ \\
Present & $385(96.3)$ \\
Duodenitis & \\
Not present & \\
Present & \\
Peptic ulcer & \\
Not present & \\
Present & \\
$\quad$ Gastric & \\
Notorinfected & \\
Infected & \\
\hline
\end{tabular}

* Data is presented as mean \pm standard deviation. The age range of the patients was $19-90$ years.

Table II. Characteristics of the patients who had positive rapid urease tests (RUTs) $(n=89)$.

\begin{tabular}{llccc}
\hline \multirow{2}{*}{$\begin{array}{l}\text { RUT results according } \\
\text { to biopsy location }\end{array}$} & \multicolumn{3}{c}{ No. of patients (\%) } \\
\cline { 3 - 5 } & $\begin{array}{c}\text { NDH } \\
(\mathbf{n}=\mathbf{5 2})\end{array}$ & $\begin{array}{c}\text { PDH } \\
(\mathbf{n}=\mathbf{3 7 )})\end{array}$ & $\begin{array}{c}\text { Total } \\
(\mathbf{n}=\mathbf{8 9})\end{array}$ \\
\hline $\begin{array}{l}\text { Gastric } \\
\text { antrum }\end{array}$ & $\begin{array}{l}\text { Gastric } \\
\text { body }\end{array}$ & & & \\
Positive & Negative & $5(9.6)$ & $7(18.9)$ & $12(13.5)$ \\
Positive & Positive & $41(78.8)$ & $19(51.4)$ & $60(67.4)$ \\
Negative & Positive & $6(11.5)$ & $11(29.7)$ & $17(19.1)$ \\
\hline
\end{tabular}

$\mathrm{NDH}$ : negative drug history; PDH: positive drug history

There was no significant difference in the distribution of the RUT results when other factors, such as previous eradication therapy $(p=0.536)$, presence of gastritis $(p=0.783)$, presence of duodenitis $(p=0.413)$, presence of gastric ulcer $(p=0.097)$ and presence of duodenal ulcer $(p=0.250)$, were analysed individually. 


\section{DISCUSSION}

RUT is the most popular biopsy-based tool for diagnosing $H$. pylori infection. It is widely used as one of the pretreatment diagnostic tests in both clinical practice and clinical trials. ${ }^{(15)}$ The simplicity, low cost and relatively rapid results of RUT make it a practical and cost-effective means of testing for $H$. pylori infection in patients who require upper endoscopy.

However, the usefulness of RUT in routine clinical practice has been compromised. Medications such as PPIs, bismuth and antibiotics, which reduce the density and/or urease activity of H. pylori, can decrease the sensitivity of RUT by up to $25 \% .{ }^{(16)}$ Such acid suppression/antibiotic therapies are highly prevalent in our local population, as evidenced by the present study, where approximately half of the patients (49.8\%) had been on such therapies within the one week prior to endoscopy. This is likely due to the changing referral patterns for $H$. pylori treatment in the last decade, with more treatments being conducted at the primary care setting. ${ }^{(17)}$

When the present study was first started in 2005, there were no firm guidelines regarding the recommended time for the administration of RUT for patients who were receiving treatment with PPIs, bismuth and/or antibiotics. Usually, patients were asked to cease such types of medications for at least one week before taking the RUT. ${ }^{(16)}$ Therefore, the cutoff time used in the present study for the definition of positive drug history was set at one week prior to testing. A more recent consensus report stated that cessation of PPIs two weeks before testing allows the bacteria to repopulate the stomach. ${ }^{(18)}$ That report also recommended that tests to determine whether $H$. pylori eradication was successful should be conducted at least four weeks after the end of the treatment (evidence level 2b). ${ }^{(18}$ However, no study has evaluated the washout period necessary after PPI treatment.

The aim of the present study was not to show when one should stop acid suppression/antibiotic therapy before RUT; our aim was to determine whether an additional biopsy is necessary in the routine use of RUT and whether acid suppression and antibiotic therapy affect RUT results. Therefore, while it is difficult to speculate how our results may have differed if the new criteria ${ }^{(18}$ for determining $\mathrm{PDH}$ and $\mathrm{NDH}$ was used, the results of the present study suggest that $H$. pylori distribution becomes patchy in the presence of a PDH (i.e. positive RUT detected only in the gastric body or only in the gastric antrum in a proportion of the patients). The present study also shows that in cases where it is not possible to stop the use of drugs 2-4 weeks prior to diagnostic gastroscopy (e.g. due to emergent or urgent indications), RUT may still be done, albeit with decreased sensitivity. This decreased sensitivity may be addressed via the use of an additional biopsy; RUT using two biopsies from different parts of the stomach (i.e. the gastric antrum and body) was shown in the present study to achieve a better yield than RUT using a single biopsy. To address the patchy distribution of $H$. pylori in the stomach after antibiotics or acid suppression therapy, it has been recommended that biopsies for RUT be obtained from two sites - the gastric antrum and gastric body. ${ }^{(9)}$
To maximise the diagnostic yield for $H$. pylori infection in gastric mucosal biopsies, some authors have recommended the use of tissue samples from at least three sites - the lesser curve angularis, the greater curve antrum and the greater curve corpus. ${ }^{(19)}$ Current guidelines recommend combining gastric antrum and gastric body biopsies for RUT if the patient has had antibiotic or PPI exposure. ${ }^{(20)} \mathrm{A}$ recent study by Moon et al found that as compared to testing tissues separately for RUT, combining tissues prior to RUT enhanced the detection of $H$. pylori by a further $8 \%$ and shortened the time needed to develop a positive reaction by approximately $50 \% .{ }^{(21)}$ However, due to time and cost constraints, some endoscopists may resort to biopsy from the gastric antrum only.

There have been conflicting reports regarding the most appropriate biopsy site for detecting $H$. pylori. While the migration of $H$. pylori from the antrum to the proximal parts of the stomach has been demonstrated among patients on acid suppression therapy with PPIs, ${ }^{(5)}$ the contrary has been shown in a study by Graham et al. (22) In Graham et al's study, H. pylori was not shown to migrate from the antrum to the corpus in response to omeprazole, thus suggesting that not much is to be gained by testing biopsy specimens from the corpus. ${ }^{(22)}$ Bermejo et al found that there was a higher proportion of positive agreement between RUT results and histology results when biopsy specimens were obtained from the gastric body, as compared to the gastric antrum. ${ }^{(23)}$ This may be due to the larger prevalence of both glandular atrophy and intestinal metaplasia at the latter site. ${ }^{(23)}$

Based on the results of the present study, acid suppression/ antibiotic therapy appears to affect the detection rate at different biopsy sites (there were differences between the $\mathrm{PDH}$ and $\mathrm{NDH}$ groups). The percentage of positive RUTs for specimens from both biopsy sites was significantly lower in the $\mathrm{PDH}$ group than in the $\mathrm{NDH}$ group (51.4\% vs. $78.8 \%$ ). This means that there was an increased incidence of cases where a positive RUT was obtained from only one biopsy site - either the gastric antrum or gastric body - in the PDH group.

While the findings of the present study support the theory that acid suppression/antibiotic therapy decreases the sensitivity of RUT, it does not provide evidence for the proximal migration of $H$. pylori to the gastric body. However, firm conclusions could not be made, as there was a small number $(n=11)$ of patients with negative RUT results from the antrum but positive RUT results from the gastric body. We also observed that there was a larger proportion of patients in the $\mathrm{PDH}$ group with positive RUT results from the antrum only. However, the number of patients in this subgroup was even smaller $(n=7)$ to be able to draw any meaningful conclusion on whether acid suppression/antibiotic therapy caused distal migration of $H$. pylori.

The results of the present study also support the recommendation of taking gastric biopsies from more than one location for RUT, so as to decrease the chance of a false negative result. We recommend a second biopsy be taken from the gastric body in patients who have a recent history of acid suppression or antibiotic therapy. In a similar study from India, testing for H. pylori using biopsy specimens from the antrum alone (i.e. no 
specimens taken from the gastric body) resulted in $8 \%$ of the patients having false negative results. ${ }^{(24)}$

This study was not without limitations. Firstly, we did not use histology to confirm the RUT results. As the sensitivity of the RUT can be significantly reduced when patients have been exposed to acid suppression or antibiotic therapy, there is a reasonable chance that some of the 162 patients with $\mathrm{PDH}$ who had negative RUTs were actually infected with $H$. pylori. In other words, there may have been an under-detection of the true incidence of $H$. pylori infection in our study population. However, this study was not designed to determine the incidence of $H$. pylori infection; it was designed to determine the usefulness of using biopsies from two separate locations for RUT in situations where histological examination for H. pylori is not routine. Secondly, the present study did not have intrasubject controls. The use of intrasubject controls would have been useful in determining whether the difference in the bacterial distribution was truly due to the effect of acid suppression/antibiotic therapy. However, based on the observed significant difference in the distribution patterns of the $\mathrm{PDH}$ and $\mathrm{NDH}$ groups, it is reasonable to conclude that acid suppression/antibiotic therapy is an important factor in the bacterial distribution of $H$. pylori. This is because it was the only factor analysed in the present study that resulted in a change in bacterial distribution. All of the other factors that were analysed did not suggest any possible link.

In conclusion, we observed a high prevalence of acid suppression or antibiotic therapy use in our study population. Patients who have a positive history for ongoing, or very recent, use of acid suppression or antibiotic therapy have significantly altered patterns of RUT results from the gastric antrum and the gastric body. In order to minimise false negative results in the detection of $H$. pylori infection, biopsies from both the gastric antrum and gastric body should be taken for RUT whenever possible.

\section{ACKNOWLEDGEMENTS}

This study was supported by a grant from the National Medical Research Council, Singapore, for the purchase of HpFast urease test kits (Reference no. NMRC/EG/CGH/2005).

\section{REFERENCES}

1. Marshall BJ, Warren JR. Unidentified curved bacilli in the stomach of patients with gastritis and peptic ulceration. Lancet 1984; 1:1311-5.

2. Wotherspoon AC, Doglioni C, Diss TC, et al. Regression of primary lowgrade $\mathrm{B}$-cell gastric lymphoma of mucosa-associated lymphoid tissue type after eradication of Helicobacter pylori. Lancet 1993; 342:575-7.
3. El-Zimaity HM. Gastric atrophy, diagnosing and staging. World J Gastroenterol 2006; 12:5757-62.

4. Mobley HL. The role of Helicobacter pylori urease in the pathogenesis of gastritis and peptic ulceration. Aliment Pharmacol Ther 1996; 10 suppl 1:57-64.

5. Logan RP, Walker MM, Misiewicz JJ, et al. Changes in the intragastric distribution of Helicobacter pylori during treatment with omeprazole. Gut 1995; 36:12-6.

6. de Boer WA, van Etten RJ, Schade RW, et al. One-day intensified lansoprazole-quadruple therapy for cure of Helicobacter pylori infection. Aliment Pharmocol Ther 1997; 11:109-12.

7. Boixeda D, Gisbet JP, de Rafael L, et al. [The importance of obtaining biopsies of the gastric body in the follow-up after eradicating treatment of Helicobacter pylori]. Med Clin (Barc) 1995; 105:566-9. Spanish.

8. Connor SJ, Seow F, Ngu MC, Katelaris PH. The effect of dosing with omeprazole on the accuracy of the 13C-urea breath test in Helicobacter pylori-infected subjects. Aliment Pharmacol Ther 1999; 13:1287-93.

9. Woo JS, el-Zimaity HM, Genta RM, Yousfi MM, Graham DY. The best gastric site for obtaining a positive rapid urease test. Helicobacter 1996; 1:256-9.

10. Weston AP, Campbell DR, Hassanein RS, et al. Prospective, multivariate evaluation of CLOtest performance. Am J Gastroenterol 1997; 92:1310-5.

11. Vassallo J, Hale R, Ahluwalia NK. CLO vs histology: optimal numbers and site of gastric biopsies to diagnose Helicobacter pylori. Eur J Gastroenterol Hepatol 2001; 13:387-90.

12. Lan HC, Chen TS, Li AF, Chang FY, Lin HC. Additional corpus biopsy enhances the detection of Helicobacter pylori infection in a background of gastritis with atrophy. BMC Gastroenterol 2012; 12:182-91.

13. Fock KM. Helicobacter pylori infection--current status in Singapore. Ann Acad Med Singapore 1997; 26:637-41.

14. Laine L, Lewin D, Naritoku W, Estrada R, Cohen H. Prospective comparison of commercially available rapid urease tests for the diagnosis of Helicobacter pylori. Gastrointest Endosc 1996; 44:523-6.

15. Ching SS, Sabanathan S, Jenkinson LR. Treatment of Helicobacter pylori in surgical practice: a randomised trial of triple versus quadruple therapy in a rural district general hospital. World J Gastroenterol 2008; 14:3855-60.

16. Midolo P, Marshall BJ. Accurate diagnosis of Helicobacter pylori. Urease tests. Gastroenterol Clin North Am 2000; 29:871-8.

17. Fock KM, Katelaris P, Sugano K, et al. Second Asia-Pacific Consensus Guidelines for Helicobacter pylori infection. J Gastroenterol Hepatol 2009; 24:1587-600.

18. Malfertheiner $\mathrm{P}$, Megraud F, O'Morain CA, et al. Management of Helicobacter pylori infection--the Maastricht IV/Florence Consensus Report. Gut 2012; 61:646-64.

19. Genta RM, Graham DY. Comparison of biopsy sites for the histopathological diagnosis of Helicobacter pylori: a topographic study of $\mathrm{H}$. pylori density and distribution. Gastrointest Endosc 1994; 40:342-5.

20. Chey WD, Wong BC. American College of Gastroenterology guideline on the management of Helicobacter pylori infection. Am J Gastroenterol 2007; 102:1808-25.

21. Moon SW, Kim TH, Kim SH, et al. United Rapid Urease Test Is Superior than Separate Test in Detecting Helicobacter pylori at the Gastric Antrum and Body Specimens. Clin Endosc 2012; 45:392-6.

22. Graham DY, Genta R, Evans DG, et al. Helicobacter pylori does not migrate from the antrum to the corpus in response to omeprazole. Am J Gastroenterol 1996; 91:2120-4.

23. Bermejo F, Boixeda D, Gisbert JP, et al. Rapid urease test utility for Helicobacter pylori infection diagnosis in gastric ulcer disease. Hepatogastroenterology 2002; 49:572-5.

24. Seth AK, Kakkar S, Manchanda GS. Role of biopsy from gastric corpus in diagnosis of Helicobacter pylori infection in patients on acid suppression therapy. MJAFI 2003; 59:216-7. 